\title{
On the Expected Number of Internal Equilibria in Random Evolutionary Games with Correlated Payoff Matrix
}

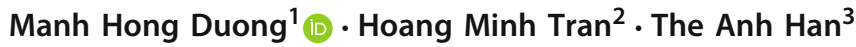

Published online: 28 July 2018

(c) The Author(s) 2018

\begin{abstract}
The analysis of equilibrium points in random games has been of great interest in evolutionary game theory, with important implications for understanding of complexity in a dynamical system, such as its behavioural, cultural or biological diversity. The analysis so far has focused on random games of independent payoff entries. In this paper, we overcome this restrictive assumption by considering multiplayer two-strategy evolutionary games where the payoff matrix entries are correlated random variables. Using techniques from the random polynomial theory, we establish a closed formula for the mean numbers of internal (stable) equilibria. We then characterise the asymptotic behaviour of this important quantity for large group sizes and study the effect of the correlation. Our results show that decreasing the correlation among payoffs (namely, of a strategist for different group compositions) leads to larger mean numbers of (stable) equilibrium points, suggesting that the system or population behavioural diversity can be promoted by increasing independence of the payoff entries. Numerical results are provided to support the obtained analytical results.
\end{abstract}

Keywords Evolutionary game theory $\cdot$ Multiplayer games $\cdot$ Replicator dynamics $\cdot$ Random polynomials $\cdot$ Number of equilibria $\cdot$ Random games

\section{Introduction}

\subsection{Motivation}

Evolutionary Game Theory (EGT) was originally introduced in 1973 by Maynard Smith and Price [41] as an application of classical game theory to biological contexts, providing

Manh Hong Duong

m.duong@imperial.ac.uk

Hoang Minh Tran

hoangtm.fami@gmail.com

The Anh Han

T.Han@tees.ac.uk

1 Department of Mathematics, Imperial College London, London SW7 2AZ, UK

2 Data Analytics Department, Esmart Systems, 1783 Halden, Norway

3 School of Computing, Media and the Arts, Teesside University, Middlesbrough TS1 3BX, UK

Birkhäuser 
explanations for odd animal behaviours in conflict situations. Since then, it has become one of the most diverse and far reaching theories in biology, finding further applications in various fields such as ecology, physics, economics and computer science $[3,9,34,35,40,45$, 49,55]. For example, in economics, it has been used to make predictions in settings where traditional assumptions about agents' rationality and knowledge may not be justified [24, 55]. In computer science, EGT has been used extensively to model dynamics and emergent behaviour in multiagent systems $[29,66]$. Furthermore, EGT has helped explain the evolution and emergence of cooperative behaviours in diverse societies, one of the most actively studied and challenging interdisciplinary problems in science $[10,35,45,46]$.

Similar to the foundational concept of Nash equilibrium in classical game theory [42], the study of equilibrium points and their stability in EGT has been of significant importance and extensive research $[4,10,12,14,15,27,28,36]$. They represent population compositions where all the strategies have the same average fitness, thus predicting the coexistence of different strategic behaviours or types in a population. The major body of such EGT literature has focused on equilibrium properties in EGT for concrete games (i.e. games with well-specified payoff structures) such as the coordination and the public goods games. For example, the maximal number of equilibria, the stability and attainability of certain equilibrium points in concrete games have been well established; see for example [4,11,50,56,62].

In contrast to the equilibrium analysis of concrete games, a recent body of works investigates random games where individual payoffs obtained from the games are randomly assigned $[10,14,15,25,27,28,36]$. This analysis has proven useful to provide answers to generic questions about a dynamical system such as its overall complexity. Using random games is useful to model and understand social and biological systems in which very limited information is available, or where the environment changes so rapidly and frequently that one cannot predict the payoffs of their inhabitants [20,26,36,39]. Moreover, even when randomly generated games are not directly representative for real-world scenarios, they are valuable as a null hypothesis that can be used to sharpen our understanding of what makes real games special [25]. In general, an important question posed in these works is what is the expected number, $E(d)$, of internal equilibria in a $d$-player game. An answer to the question provides important insights into the understanding of the expected levels of behavioural diversity or biodiversity one can expect in a dynamical system [27,38,64]. It would allow us to predict the level of biodiversity in multiplayer interactions, describing the probability of which a certain state of biodiversity may occur. Moreover, computing $E(d)$ provides useful upperbounds for the probability $p_{m}$ that a certain number $m$ of equilibria is attainted, since [36]: $p_{m} \leq E(d) / m$. Of particular interest is such an estimate for the probability of attaining the maximal of internal equilibria, i.e. $p_{d-1}$, as in the Feldman-Karlin conjecture [2].

Mathematically, to find internal equilibria in a $d$-player game with two strategies $A$ and $B$, one needs to solve the following polynomial equation for $y>0$ (see Eq. 5 and its derivation in Sect. 2),

$$
P(y):=\sum_{k=0}^{d-1} \beta_{k}\left(\begin{array}{c}
d-1 \\
k
\end{array}\right) y^{k}=0,
$$

where $\beta_{k}=a_{k}-b_{k}$, with $a_{k}$ and $b_{k}$ being random variables representing the payoff entries of the game payoff matrix for $A$ and $B$, respectively. Therefore, calculating $E(d)$ amounts to the computation of the expected number of positive zeros of the (random) polynomial $P$. As will be shown in Sect. 2, the set of positive roots of $P$ is the same as that of the so-called gain function which is a Bernstein polynomial. Thus, one can gain information about internal equilibria of a multiplayer game via studying positive roots of Bernstein polynomials. For 
deterministic multiplayer games, this has already been carried out in the literature [48]. One of the main goals of this paper is to extend this research to random multiplayer games via studying random polynomials.

In $[27,28,36]$, the authors provide both numerical and analytical results for games with a small number of players $(d \leq 4)$, focusing on the probability of attaining a maximal number of equilibrium points. These works use a direct approach by solving Equation (1), expressing the positivity of its zeros as domains of conditions for the coefficients and then integrating over these domains to obtain the corresponding probabilities. However, in general, a polynomial of degree five or higher is not analytically solvable [1]. Therefore, the direct approach cannot be generalised to larger $d$. More recently, in $[14,15]$ the authors introduce a novel method using techniques from random polynomials to calculate $E(d)$ with an arbitrary $d$, under the assumption that the entries of the payoff matrix are independent normal random variables. More precisely, they derive a computationally implementable formula for $E(d)$ for arbitrary $d$ and prove the following monotonicity and asymptotic behaviour of $E(d)$ :

$$
\frac{E(d)}{d-1} \text { is decreasing and } \lim _{d \rightarrow \infty} \frac{\ln E(d)}{\ln (d-1)}=\frac{1}{2} .
$$

However, the requirement that the entries of the payoff matrix are independent random variables is rather restricted from both mathematical and biological points of view. In evolutionary game theory, correlations may arise in various scenarios particularly when there are environmental randomness and interaction uncertainty such as in games of cyclic dominance [59], coevolutionary multigames [58] or when individual contributions are correlated to the surrounding contexts (e.g. due to limited resource) [60], see also recent reviews [16,57] for more examples. One might expect some strategies to have many similar properties and hence yield similar results for a given response of the respective opponent [5]. Furthermore, in a multiplayer game (such as the public goods games and their generalisations), a strategy's payoffs, which may differ for different group compositions, can be expected to be correlated given a specific nature of the strategy [30-33,47,64]. Similarly, different strategies' payoffs may be correlated given the same group composition. From a mathematical perspective, the study of real zeros of random polynomials with correlated coefficients has attracted substantial attention, see e.g. [13,21-23,51].

In this paper, we remove the assumption on the dependence of the coefficients. We will study the expected number of internal equilibria and its various properties for random evolutionary games in which the entries of the payoff matrix are correlated random variables.

\subsection{Summary of Main Results}

We now summarise the main results of this paper. More detailed statements will be presented in the sequel sections. We consider $d$-player two-strategy random games in which the coefficients $\beta_{k}(k \in\{0, \ldots, d-1\})$ can be correlated random variables, satisfying that $\operatorname{corr}\left(\beta_{i}, \beta_{j}\right)=r$ for $i \neq j$ and for some $0 \leq r \leq 1$ (see Lemma 1 about this assumption).

The main result of the paper is the following theorem which provides a formula for the expected number, $E(r, d)$, of internal equilibria, characterises its asymptotic behaviour and studies the effect of the correlation.

Theorem 1 (On the expected number of internal equilibria)

(1) (Computational formula for $E(r, d)$ )

$$
E(r, d)=2 \int_{0}^{1} f(t ; r, d) d t,
$$


where the density function $f(t ; r, d)$ is given explicitly in (8).

(2) (Monotonicity of $E(r, d)$ with respect to $r$ ) The function $r \mapsto E(r, d)$ decreases for any given $d$.

(3) (Asymptotic behaviour of $E(r, d)$ for large d) We perform formal asymptotic computations to get

$$
E(r, d)\left\{\begin{array}{l}
\sim \frac{\sqrt{2 d-1}}{2} \sim \mathcal{O}\left(d^{1 / 2}\right) \text { if } r=0, \\
\sim \frac{d^{1 / 4}(1-r)^{1 / 2}}{2 \pi^{5 / 4} r^{1 / 2}} \frac{8 \Gamma\left(\frac{5}{4}\right)^{2}}{\sqrt{\pi}} \sim \mathcal{O}\left(d^{1 / 4}\right) \text { if } 0<r<1, \\
=0 \quad \text { if } r=1 .
\end{array}\right.
$$

We compare this asymptotic behaviour numerically with the analytical formula obtained in part 1 .

This theorem clearly shows that the correlation $r$ has a significant effect on the expected number of internal equilibria $E(r, d)$. For sufficiently large $d$, when $r$ increases from 0 (uncorrelated) to 1 (identical), $E(r, d)$ reduces from $\mathcal{O}\left(d^{1 / 2}\right)$ at $r=0$, to $\mathcal{O}\left(d^{1 / 4}\right)$ for $0<r<1$ and to 0 at $r=1$. This theorem generalises and improves the main results in [15] for the case $r=0$ : the asymptotic behaviour, $E(r, d) \sim \frac{\sqrt{2 d-1}}{2}$, is stronger than (2). In addition, as a by-product of our analysis, we provide an asymptotic formula for the expected number of real zeros of a random Bernstein polynomial as conjectured in [18], see Sect. 6.7.

\subsection{Methodology of the Present Work}

We develop further the connections between EGT and random/deterministic polynomials theory discovered in $[14,15]$. The integral representation (3) is derived from the theory of [19], which provides a general formula for the expected number of real zeros of a random polynomial in a given domain, and the symmetry of the game, see Theorem 2; the monotonicity and asymptotic behaviour of $E(r, d)$ are obtained by using connections to Legendre polynomials, which were described in [15], see Theorems 3 and 1.

\subsection{Organisation of the Paper}

The rest of the paper is organised as follows. In Sect. 2, we recall the replicator dynamics for multiplayer two-strategy games. In Sect. 3, we prove and numerically validate the first and the second parts of Theorem 1. Section 4 is devoted to the proof of the last part of Theorem 1 and its numerical verification. Section 5 provides further discussion, and finally, "Appendix 6" contains detailed computations and proofs of technical results.

\section{Replicator Dynamics}

A fundamental model of evolutionary game theory is the replicator dynamics $[35,45,63,65$, 69], describing that whenever a strategy has a fitness larger than the average fitness of the population, it is expected to spread. From the replicator dynamics, one then can derive a polynomial equation that an internal equilibrium of a multiplayer game satisfies. To this end, we consider an infinitely large population with two strategies, A and B. Let $x, 0 \leq x \leq 1$, be the frequency of strategy $\mathrm{A}$. The frequency of strategy $\mathrm{B}$ is thus $(1-x)$. The interaction of the individuals in the population is in randomly selected groups of $d$ participants, that 
is, they play and obtain their fitness from $d$-player games. The game is defined through a $(d-1)$-dimensional payoff matrix [27], as follows. Let $a_{k}$ (respectively, $b_{k}$ ) be the payoff of an A strategist (respectively, a B strategist) obtained when interacting with a group of $d-1$ other players containing $k$ A strategists (i.e. $d-1-k$ B strategists). In this paper, we consider symmetric games where the payoffs do not depend on the ordering of the players. Asymmetric games will be studied in a forthcoming paper. In the symmetric case, the average payoffs of $A$ and $B$ are, respectively

$$
\pi_{A}=\sum_{k=0}^{d-1} a_{k}\left(\begin{array}{c}
d-1 \\
k
\end{array}\right) x^{k}(1-x)^{d-1-k}, \quad \pi_{B}=\sum_{k=0}^{d-1} b_{k}\left(\begin{array}{c}
d-1 \\
k
\end{array}\right) x^{k}(1-x)^{d-1-k} .
$$

Internal equilibria are those points that satisfy the condition that the fitnesses of both strategies are the same $\pi_{A}=\pi_{B}$, which gives rise to $g(x)=0$ where $g(x)$ is the so-called gain function given by $[6,48]$

$$
g(x)=\sum_{k=0}^{d-1} \beta_{k}\left(\begin{array}{c}
d-1 \\
k
\end{array}\right) x^{k}(1-x)^{d-1-k},
$$

where $\beta_{k}=a_{k}-b_{k}$. Note that this equation can also be derived from the definition of an evolutionary stable strategy (ESS), see, e.g. [4]. As also discussed in that paper, the evolutionary solution of the game (such as the set of ESSs or the set of stable rest points of the replicator dynamics) involves not only finding the roots of the gain function $g(x)$ but also determining the behaviour of $g(x)$ in the vicinity of such roots. We also refer the reader to $[65,69]$ and references therein for further discussion on relations between ESSs and game dynamics. Using the transformation $y=\frac{x}{1-x}$, with $0<y<+\infty$, and dividing $g(x)$ by $(1-x)^{d-1}$, we obtain the following polynomial equation for $y$

$$
P(y):=\sum_{k=0}^{d-1} \beta_{k}\left(\begin{array}{c}
d-1 \\
k
\end{array}\right) y^{k}=0 .
$$

As in [14,15,27], we are interested in random games where $a_{k}$ and $b_{k}$ (thus $\beta_{k}$ ), for $0 \leq k \leq$ $d-1$, are random variables. However, in contrast to these papers where $\beta_{k}$ are assumed to be independent, we analyse here a more general case where they are correlated. In particular, we consider that any pair $\beta_{i}$ and $\beta_{j}$, with $0 \leq i \neq j \leq d-1$, have a correlation $r(0 \leq r \leq 1)$. In general, $r=0$ means $\beta_{i}$ and $\beta_{j}$ are independent, while when $r=1$ they have a (perfectly) linear correlation, and the larger $r$ is the stronger they are correlated. It is noteworthy that this type of dependency between the coefficients is common in the literature on evolutionary game theory $[5,25]$ as well as random polynomial theory $[13,23,51]$.

The next lemma shows how this assumption arises naturally from simple assumptions on the game payoff entries. To state the lemma, let $\operatorname{cov}(X, Y)$ and $\operatorname{corr}(X, Y)$ denote the covariance and correlation between random variables $X$ and $Y$, respectively; moreover, $\operatorname{var}(X)=\operatorname{cov}(X, X)$ denotes the variance of $X$.

Lemma 1 Suppose that, for $0 \leq i \neq j \leq d-1$,

$-\operatorname{var}\left(a_{i}\right)=\operatorname{var}\left(b_{i}\right)=\eta^{2}$

$-\operatorname{corr}\left(a_{i}, a_{j}\right)=r_{a}, \operatorname{corr}\left(b_{i}, b_{j}\right)=r_{b}$,

$-\operatorname{corr}\left(a_{i}, b_{j}\right)=r_{a b}, \operatorname{corr}\left(a_{i}, b_{i}\right)=r_{a b}^{\prime}$.

Then, the correlation between $\beta_{i}$ and $\beta_{j}$, for $1 \leq i \neq j \leq d-1$, is given by

$$
\operatorname{corr}\left(\beta_{i}, \beta_{j}\right)=\frac{r_{a}+r_{b}-2 r_{a b}}{2\left(1-r_{a b}^{\prime}\right)}
$$


which is a constant. Clearly, it increases with $r_{a}, r_{b}$ and $r_{a b}^{\prime}$ while decreasing with $r_{a b}$. Moreover, if $r_{a}+r_{b}=2 r_{a b}$, then $\beta_{i}$ and $\beta_{j}$ are independent. Also, if $r_{a b}=r_{a b}^{\prime}=0$, i.e. when payoffs from different strategists are independent, we have: $\operatorname{corr}\left(\beta_{i}, \beta_{j}\right)=\frac{r_{a}+r_{b}}{2}$. If we further assume that $r_{a}=r_{b}=r$, then $\operatorname{corr}\left(\beta_{i}, \beta_{j}\right)=r$.

Proof See "Appendix 6.1".

The assumptions in Lemma 1 mean that a strategist's payoffs for different group compositions have a constant correlation, which in general is different from the cross-correlation of payoffs for different strategists. These assumptions arise naturally for example in a multiplayer game (such as the public goods games and their generalisations), since a strategist's payoffs, which may differ for different group compositions, can be expected to be correlated given a specific nature of the strategy (e.g. cooperative vs. defective strategies in the public goods games). These natural assumptions regarding payoffs' correlations are just to ensure the pairs $\beta_{i}$ and $\beta_{j}, 0 \leq i \neq j \leq d-1$, have a constant correlation. Characterising the general case where $\beta_{i}$ and $\beta_{j}$ have varying correlations would be mathematically interesting but is out of the scope of this paper. We will discuss further this issue particularly for other types of correlations in Sect. 5.

\section{The Expected Number of Internal Equilibria $E(r, d)$}

We consider the case where $\beta_{k}$ are standard normal random variables but assume that all the pairs $\beta_{i}$ and $\beta_{j}$, for $0 \leq i \neq j \leq d-1$, have the same correlation $0 \leq r \leq 1$ (cf. Lemma 1 ).

In this section, we study the expected number of internal equilibria $E(r, d)$. The starting point of the analysis of this section is an improper integral to compute $E(r, d)$ as a direct application of the Edelman-Kostlan theorem [19], see Lemma 2. We then further simplify this formula to obtain a more computationally tractable one (see Theorem 2 ) and then prove a monotone property of $E(r, d)$ as a function of the correlation $r$, see Theorem 3 .

\subsection{Computations of $E(r, d)$}

Lemma 2 Assume that $\beta_{k}$ are standard normal random variables and that for any $i \neq j$, the correlation between $\beta_{i}$ and $\beta_{j}$ is equal to $r$ for some $0 \leq r \leq 1$. Then, the expected number of internal equilibria, $E(r, d)$, in a d-player random game with two strategies is given by

$$
E(r, d)=\int_{0}^{\infty} f(t ; r, d) d t
$$

where

$$
\begin{aligned}
{[\pi f(t ; r, d)]^{2}=} & \frac{(1-r) \sum_{i=0}^{d-1} i^{2}\left(\begin{array}{c}
d-1 \\
i
\end{array}\right)^{2} t^{2(i-1)}+r(d-1)^{2}(1+t)^{2(d-2)}}{(1-r) \sum_{i=0}^{d-1}\left(\begin{array}{c}
d-1 \\
i
\end{array}\right)^{2} t^{2 i}+r(1+t)^{2(d-1)}} \\
& -\left[\frac{(1-r) \sum_{i=0}^{d-1} i\left(\begin{array}{c}
d-1 \\
i
\end{array}\right)^{2} t^{2 i-1}+r(d-1)(1+t)^{2 d-3}}{(1-r) \sum_{i=0}^{d-1}\left(\begin{array}{c}
d-1 \\
i
\end{array}\right)^{2} t^{2 i}+r(1+t)^{2(d-1)}}\right]^{2} .
\end{aligned}
$$


Proof According to [19] (see also [14,15]), we have

$$
E(r, d)=\int_{0}^{\infty} f(t ; r, d) \mathrm{d} t,
$$

where the density function $f(t ; r, d)$ is determined by

$$
f(t ; r, d)=\frac{1}{\pi}\left[\left.\frac{\partial^{2}}{\partial x \partial y}\left(\log v(x)^{T} \mathcal{C} v(y)\right)\right|_{y=x=t}\right]^{\frac{1}{2}},
$$

with the covariance matrix $\mathcal{C}$ and the vector $v$ given by

$$
\mathcal{C}_{i j}=\left\{\begin{array}{l}
\left(\begin{array}{c}
d-1 \\
i
\end{array}\right)^{2}, \quad \text { if } i=j \\
r\left(\begin{array}{c}
d-1 \\
i
\end{array}\right)\left(\begin{array}{c}
d-1 \\
j
\end{array}\right), \quad \text { if } i \neq j .
\end{array} \quad \text { and } \quad v(x)=\left(\begin{array}{c}
1 \\
x \\
\vdots \\
x^{d-1}
\end{array}\right) .\right.
$$

Let us define

$$
\begin{aligned}
H(x, y) & :=v(x)^{T} \mathcal{C} v(y) \\
& =\sum_{i=0}^{d-1}\left(\begin{array}{c}
d-1 \\
i
\end{array}\right)^{2} x^{i} y^{i}+r \sum_{i \neq j=0}^{d-1}\left(\begin{array}{c}
d-1 \\
i
\end{array}\right)\left(\begin{array}{c}
d-1 \\
j
\end{array}\right) x^{i} y^{j} \\
& =(1-r) \sum_{i=0}^{d-1}\left(\begin{array}{c}
d-1 \\
i
\end{array}\right)^{2} x^{i} y^{i}+r\left(\sum_{i=0}^{d-1}\left(\begin{array}{c}
d-1 \\
i
\end{array}\right) x^{i}\right)\left(\sum_{j=0}^{d-1}\left(\begin{array}{c}
d-1 \\
j
\end{array}\right) y^{j}\right) .
\end{aligned}
$$

Then, we compute

$$
\frac{\partial^{2}}{\partial_{x} \partial_{y}}\left(\log v(x)^{T} \mathcal{C} v(y)\right)=\frac{\partial^{2}}{\partial_{x} \partial_{y}} \log H(x, y)=\frac{\partial_{x y}^{2} H(x, y)}{H(x, y)}-\frac{\partial_{x} H(x, y) \partial_{y} H(x, y)}{H(x, y)^{2}} .
$$

Particularly, for $y=x=t$, we obtain

$$
\begin{aligned}
\left.\frac{\partial^{2}}{\partial_{x} \partial_{y}}\left(\log v(x)^{T} \mathcal{C} v(y)\right)\right|_{y=x=t} & =\left.\left(\frac{\partial_{x y}^{2} H(x, y)}{H(x, y)}-\frac{\partial_{x} H(x, y) \partial_{y} H(x, y)}{H(x, y)^{2}}\right)\right|_{y=x=t} \\
& =\frac{\left.\partial_{x y}^{2} H(x, y)\right|_{y=x=t}}{H(t, t)}-\left(\frac{\left.\partial_{x} H(x, y)\right|_{y=x=t}}{H(t, t)}\right)^{2} .
\end{aligned}
$$

Using (11), we can compute each term on the right-hand side of the above expression explicitly

$$
\begin{aligned}
& H(t, t)=(1-r) \sum_{i=0}^{d-1}\left(\begin{array}{c}
d-1 \\
i
\end{array}\right)^{2} t^{2 i}+r\left(\sum_{i=0}^{d-1}\left(\begin{array}{c}
d-1 \\
i
\end{array}\right) t^{i}\right)^{2}, \\
& \left.\partial_{x} H(x, y)\right|_{y=x=t}=(1-r) \sum_{i=0}^{d-1} i\left(\begin{array}{c}
d-1 \\
i
\end{array}\right)^{2} t^{2 i-1} \\
& \quad+r\left(\sum_{i=0}^{d-1} i\left(\begin{array}{c}
d-1 \\
i
\end{array}\right) t^{i}\right)\left(\sum_{j=0}^{d-1}\left(\begin{array}{c}
d-1 \\
j
\end{array}\right) t^{j-1}\right),
\end{aligned}
$$




$$
\left.\partial_{x y}^{2} H(x, y)\right|_{y=x=t}=(1-r) \sum_{i=0}^{d-1} i^{2}\left(\begin{array}{c}
d-1 \\
i
\end{array}\right)^{2} t^{2(i-1)}+r\left(\sum_{i=0}^{d-1} i\left(\begin{array}{c}
d-1 \\
i
\end{array}\right) t^{i-1}\right)^{2} .
$$

We can simplify further the above expressions using the following computations which are attained from the binomial theorem and its derivatives

$$
\begin{aligned}
& \left(\sum_{i=0}^{d-1}\left(\begin{array}{c}
d-1 \\
i
\end{array}\right) t^{i}\right)^{2}=(1+t)^{2(d-1)} \\
& \left(\sum_{i=0}^{d-1} i\left(\begin{array}{c}
d-1 \\
i
\end{array}\right) t^{i-1}\right)^{2}=\left(\frac{d}{\mathrm{~d} t} \sum_{i=0}^{d-1}\left(\begin{array}{c}
d-1 \\
i
\end{array}\right) t^{i}\right)^{2}=\left(\frac{d}{\mathrm{~d} t}(1+t)^{d-1}\right)^{2} \\
& =(d-1)^{2}(1+t)^{2(d-2)}, \\
& \left(\sum_{i=0}^{d-1} i\left(\begin{array}{c}
d-1 \\
i
\end{array}\right) t^{i}\right)\left(\sum_{j=0}^{d-1}\left(\begin{array}{c}
d-1 \\
j
\end{array}\right) t^{j-1}\right)=\frac{1}{2} \frac{d}{\mathrm{~d} t}\left(\sum_{i=0}^{d-1}\left(\begin{array}{c}
d-1 \\
i
\end{array}\right) t^{i}\right)^{2} \\
& =\frac{1}{2} \frac{d}{\mathrm{~d} t}(1+t)^{2(d-1)}=(d-1)(1+t)^{2 d-3} .
\end{aligned}
$$

Substituting (12) and (13) back into (9), we obtain (8) and complete the proof.

Next, we will show that, as in the case $r=0$ studied in $[14,15]$, the improper integral (7) can be reduced to a definite integral from 0 to 1 . A crucial property enables us to do so is the symmetry of the strategies. The main result of this section is the following theorem (cf. Theorem 1-(1)).

Theorem 2 (1) The density function $f(t ; r, d)$ satisfies that

$$
f(1 / t ; r, d)=t^{2} f(t ; r, d) .
$$

(2) (Computable formula for $E(r, d)$ ). $E(r, d)$ can be computed via

$$
E(r, d)=2 \int_{0}^{1} f(t) \mathrm{d} t=2 \int_{1}^{\infty} f(t) \mathrm{d} t .
$$

Proof The proof of the first part is lengthy and is given in "Appendix 6.2". Now, we prove the second part. We have

$$
E(r, d)=\int_{0}^{\infty} f(t ; r, d) \mathrm{d} t=\int_{0}^{1} f(t ; r, d) \mathrm{d} t+\int_{1}^{\infty} f(t ; r, d) \mathrm{d} t .
$$

By changing of variables $t:=\frac{1}{s}$, the first integral on the right-hand side of (16) can be transformed as

$$
\int_{0}^{1} f(t ; r, d) \mathrm{d} t=\int_{1}^{\infty} f(1 / s ; r, d) \frac{1}{s^{2}} \mathrm{~d} s=\int_{1}^{\infty} f(s ; r, d) \mathrm{d} s,
$$

where we have used (14) to obtain the last equality. The assertion (15) is then followed from (16) and (17).

As in [15], we can interpret the first part of Theorem 2 as a symmetric property of the game. We recall that $t=\frac{y}{1-y}$, where $y$ and $1-y$ are, respectively, the fractions of strategies 1 and 
2. We write the density function $f(t ; r, d)$ in terms of $y$ using the change of variable formula as follows.

$$
f(t ; r, d) \mathrm{d} t=f\left(\frac{y}{1-y} ; r, d\right) \frac{1}{(1-y)^{2}} d y:=g(y ; r, d) d y,
$$

where

$$
g(y ; r, d):=f\left(\frac{y}{1-y} ; r, d\right) \frac{1}{(1-y)^{2}} .
$$

The following lemma expresses the symmetry of the strategies. (Swapping the index labels converts an equilibrium at $y$ to one at $1-y$.)

Corollary 1 The function $y \mapsto g(y ; r, d)$ is symmetric about the line $y=\frac{1}{2}$, i.e.

$$
g(y ; r, d)=g(1-y ; r, d) .
$$

Proof The equality (19) is a direct consequence of (14). We have

$$
\begin{aligned}
g(1-y ; r, d)=f\left(\frac{1-y}{y} ; r, d\right) \frac{1}{y^{2}} & \stackrel{(14)}{=} f\left(\frac{y}{1-y} ; r, d\right) \frac{y^{2}}{(1-y)^{2}} \frac{1}{y^{2}} \\
& =f\left(\frac{y}{1-y} ; r, d\right) \frac{1}{(1-y)^{2}}=g(y ; r, d) .
\end{aligned}
$$

\subsection{Monotonicity of $r \mapsto E(r, d)$}

In this section, we study the monotone property of $E(r, d)$ as a function of the correlation $r$. The main result of this section is the following theorem on the monotonicity of $r \mapsto E(r, d)$ (cf. Theorem 1-(2)).

Theorem 3 The function $r \mapsto f(t ; r, d)$ is decreasing. As a consequence, $r \mapsto E(r, d)$ is also decreasing.

Proof We define the following notations:

$$
\begin{aligned}
& M_{1}=M_{1}(t ; r, d)=\sum_{i=0}^{d-1}\left(\begin{array}{c}
d-1 \\
i
\end{array}\right)^{2} t^{2 i}, \quad M_{2}=M_{2}(t ; r, d)=(1+t)^{2(d-1)}, \\
& A_{1}=A_{1}(t ; r, d)=\sum_{i=0}^{d-1} i^{2}\left(\begin{array}{c}
d-1 \\
i
\end{array}\right)^{2} t^{2(i-1)}, \quad A_{2}=A_{2}(t ; r, d)=(d-1)^{2}(1+t)^{2(d-2)}, \\
& B_{1}=B_{1}(t ; r, d)=\sum_{i=0}^{d-1} i\left(\begin{array}{c}
d-1 \\
i
\end{array}\right)^{2} t^{2 i-1}, \quad B_{2}=B_{2}(t ; r, d)=(d-1)(1+t)^{2 d-3}, \\
& M=(1-r) M_{1}+r M_{2}, \quad A=(1-r) A_{1}+r A_{2}, \quad B=(1-r) B_{1}+r B_{2} .
\end{aligned}
$$

Then, the density function $f(t ; r, d)$ in $(8)$ can be written as

$$
(\pi f(t ; r, d))^{2}=\frac{A M-B^{2}}{M^{2}} .
$$


Taking the derivation with respect to $r$ of the right-hand side of (20), we obtain

$$
\begin{aligned}
\frac{\partial}{\partial r} & \left(\frac{A M-B^{2}}{M^{2}}\right) \\
& =\frac{\left(A^{\prime} M+M^{\prime} A-2 B B^{\prime}\right) M^{2}-2\left(A M-2 B^{2}\right) M M^{\prime}}{M^{4}} \\
& =\frac{\left(A^{\prime} M+M^{\prime} A-2 B B^{\prime}\right) M-2\left(A M-B^{2}\right) M^{\prime}}{M^{3}} \\
& =\frac{2 B\left(B M^{\prime}-B^{\prime} M\right)-M^{\prime}\left(A M^{\prime}-M A^{\prime}\right)}{M^{3}} \\
& \stackrel{(*)}{=} \frac{2 B\left(B_{1} M_{2}-M_{1} B_{2}\right)-M\left(A_{1} M_{2}-M_{1} A_{2}\right)}{M^{3}} \\
& =\frac{2 B\left(B_{1}(1+t)^{2(d-1)}-M_{1}(d-1)(1+t)^{2 d-3}\right)-M\left(A_{1}(1+t)^{2(d-1)}-M_{1}(d-1)^{2}(1+t)^{2(d-2)}\right)}{M^{3}} \\
& =\frac{(1+t)^{2 d-4}\left\{2(t+1) B\left[B_{1}(1+t)-M_{1}(d-1)\right]-M\left[A_{1}(1+t)^{2}-M_{1}(d-1)^{2}\right]\right\}}{M^{3}} .
\end{aligned}
$$

Note that to obtain $(*)$ above we have used the following simplifications

$$
\begin{aligned}
B M^{\prime}-B^{\prime} M & =\left[B_{1}+r\left(B_{2}-B_{1}\right)\right]\left(M_{2}-M_{1}\right)-\left(B_{2}-B_{1}\right)\left[M_{1}+r\left(M_{2}-M_{1}\right)\right] \\
& =B_{1}\left(M_{2}-M_{1}\right)-\left(B_{2}-B_{1}\right) M_{1} \\
& =B_{1} M_{2}-M_{1} B_{2},
\end{aligned}
$$

and similarly,

$$
A M^{\prime}-A^{\prime} M=A_{1} M_{2}-M_{1} A_{2} .
$$

Since $M>0$ and according to Proposition 2,

$$
2(t+1) B\left[B_{1}(1+t)-M_{1}(d-1)\right]-M\left[A_{1}(1+t)^{2}-M_{1}(d-1)^{2}\right] \leq 0,
$$

it follows that

$$
\frac{\partial}{\partial r}\left(\frac{A M-B^{2}}{M^{2}}\right) \leq 0 .
$$

The assertion of the theorem is then followed from this and (20).

As a consequence, we can derive the monotonicity property of the number of stable equilibrium points, denoted by $S E(r, d)$. It is based on the following property of stable equilibria in multiplayer two-strategy evolutionary games, which has been proved in [36, Theorem 3] for payoff matrices with independent entries. We provide a similar proof below for matrices with exchangeable payoff entries. We need the following auxiliary lemma whose proof is presented in "Appendix 6.3".

Lemma 3 Let $X$ and $Y$ be two exchangeable random variables, i.e. their joint probability distribution $f_{X, Y}(x, y)$ is symmetric, $f_{X, Y}(x, y)=f_{X, Y}(y, x)$. Then, $Z=X-Y$ is symmetrically distributed about 0 , i.e. its probability distribution satisfies $f_{Z}(z)=f_{Z}(-z)$. In addition, if $X$ and $Y$ are iid, then they are exchangeable.

Theorem 4 Suppose that $a_{k}$ and $\beta_{k}$ are exchangeable random variables. For d-player evolutionary games with two strategies, the following holds

$$
S E(r, d)=\frac{1}{2} E(r, d) .
$$


Proof The replicator equation in this game is given by [27,32]

$$
\dot{x}=x(1-x) \sum_{k=0}^{d-1} \beta_{k}\left(\begin{array}{c}
d-1 \\
k
\end{array}\right) x^{k}(1-x)^{d-1-k} .
$$

Suppose $x^{*} \in(0,1)$ is an internal equilibrium of the system and $h(x)$ is the polynomial on the right-hand side of the equation. Since $x^{*}$ is stable if and only if $h^{\prime}\left(x^{*}\right)<0$ which can be simplified to [36]

$$
\sum_{k=1}^{d-1} k \beta_{k}\left(\begin{array}{c}
d-1 \\
k
\end{array}\right) y^{* k-1}<0,
$$

where $y^{*}=\frac{x^{*}}{1-x^{*}}$. As a system admits the same set of equilibria if we change the sign of all $\beta_{k}$ simultaneously, and for such a change the above inequality would change the direction (thus the stable equilibrium $x^{*}$ would become unstable), all we need to show for the theorem to hold is that $\beta_{k}$ has a symmetric density function. This is guaranteed by Lemma 3 since $\beta_{k}=a_{k}-b_{k}$ where $a_{k}$ and $b_{k}$ are exchangeable.

Corollary 2 Under the assumption of Theorem 4, the expected number of stable equilibrium points $S E(r, d)$ is a decreasing function with respect to $r$.

Proof This is a direct consequence of Theorems 3 and 4 .

\subsection{Monotonicity of $E(r, d)$ : Numerical Investigation}

In this section, we numerically validate the analytical results obtained in the previous section. In Fig. 1, we plot the functions $r \mapsto E(r, d)$ for several values of $d$ (left panel) and $d \mapsto$ $E(r, d)$ for different values of $r$ using formula 7 (right panel). In the panel on the left, we also show the value of $E(r, d)$ obtained from samplings. That is, we generate $10^{6}$ samples of $\beta_{k}(0 \leq k \leq d-1)$ where $\beta_{k}$ are normally distributed random variables satisfying that $\operatorname{corr}\left(\beta_{i}, \beta_{j}\right)=r$ for $0 \leq i \neq j \leq d-1$. For each sample, we solve Eq. (5) to obtain the corresponding number internal equilibria (i.e. the number of positive zeros of the polynomial equation). By averaging over all the $10^{6}$ samples, we obtain the probability of observing $m$ internal equilibria, $\bar{p}_{m}$, for each $0 \leq m \leq d-1$. Finally, the mean or expected number of internal equilibria is calculated as $E(r, d)=\sum_{m=0}^{d-1} m \cdot \bar{p}_{m}$. The figure shows the agreement of results obtained from analytical and sampling methods. In addition, it also demonstrates the decreasing property of $r \mapsto E(r, d)$, which was proved in Theorem 3. Additionally, we observe that $E(r, d)$ increases with the group size, $d$.

Note that to generate correlated normal random variables, we use the following algorithm that can be found in many textbooks, for instance [43, Section 4.1.8].

Algorithm 5 Generate $n$ correlated Gaussian distributed random variables $\mathbf{Y}=\left(Y_{1}, \ldots, Y_{n}\right)$, $\mathbf{Y} \sim \mathcal{N}(\mu, \Sigma)$, given the mean vector $\mu$ and the covariance matrix $\Sigma$.

Step 1. Generate a vector of uncorrelated Gaussian random variables, $\mathbf{Z}$,

Step 2. Define $\mathbf{Y}=\mu+C \mathbf{Z}$ where $C$ is the square root of $\Sigma\left(\right.$ i.e. $\left.C C^{T}=\Sigma\right)$.

The square root of a matrix can be found using the Cholesky decomposition. These two steps are easily implemented in Mathematica. 

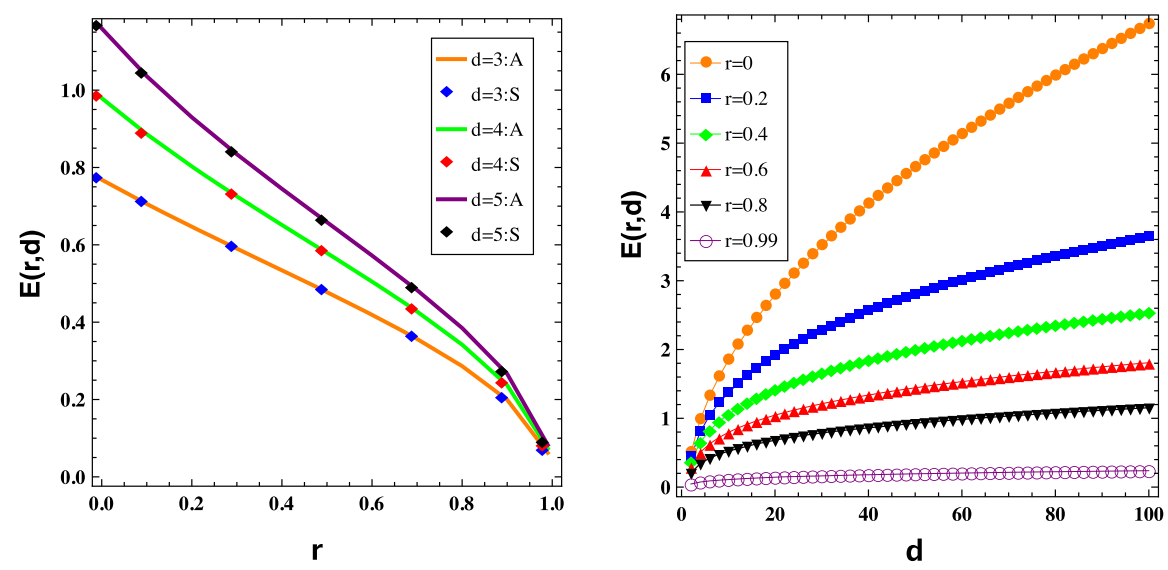

Fig. 1 (Left) Plot of $r \mapsto E(r, d)$ for different values of $d$. The solid lines are generated from analytical (A) formulas of $E(r, d)$ as defined in Eq. (7). The solid diamonds capture simulation (S) results obtained by averaging over $10^{6}$ samples of $\beta_{k}(1 \leq k \leq d-1)$, where these $\beta_{k}$ are correlated, normally standard random variables. To generate correlated random variables, the algorithm described in Algorithm 5 was used. (Right) Plot of $d \mapsto E(r, d)$ for different values of $r$. We observe that $E(r, d)$ decreases with respect to $r$ but increases with respect to $d$

\section{Asymptotic Behaviour of $E(r, d)$}

\subsection{Asymptotic Behaviour of $E(r, d)$ : Formal Analytical Computations}

In this section we perform formal asymptotic analysis to understand the behaviour of $E(r, d)$ when $d$ becomes large.

Proposition 1 We have the following asymptotic behaviour of $E(r, d)$ as $d \rightarrow \infty$

$$
E(r, d)\left\{\begin{array}{l}
\sim \frac{\sqrt{2 d-1}}{2} \text { if } r=0, \\
\sim \frac{d^{1 / 4}(1-r)^{1 / 2}}{2 \pi^{5 / 4} r^{1 / 2}} \frac{8 \Gamma\left(\frac{5}{4}\right)^{2}}{\sqrt{\pi}} \text { if } 0<r<1, \\
=0 \quad \text { if } r=1 .
\end{array}\right.
$$

Proof We consider the case $r=1$ first. In this case, we have

$$
\begin{aligned}
& M(t)=M_{2}(t)=(1+t)^{2(d-1)}, A(t)=A_{2}(t)=(d-1)^{2}(1+t)^{2(d-2)}, \\
& B(t)=B_{2}(t)=(d-1)(1+t)^{2 d-3} .
\end{aligned}
$$

Since $A_{2}(t) M_{2}(t)-B_{2}^{2}(t)=0$, we obtain $f(t ; 1, d)=0$. Therefore $E(1, d)=0$.

We now deal with the case $0 \leq r<1$. According to [7, Example 2, page 229], [68], for any $x>1$

$$
P_{d}(x)=\frac{1}{\sqrt{2 d \pi}} \frac{\left(x+\sqrt{x^{2}-1}\right)^{d+1 / 2}}{\left(x^{2}-1\right)^{1 / 4}}+\mathcal{O}\left(d^{-1}\right) \text { as } d \rightarrow \infty .
$$


Therefore,

$$
\begin{aligned}
& M_{1}=\left(1-t^{2}\right)^{d-1} P_{d-1}\left(\frac{1+t^{2}}{1-t^{2}}\right) \sim \frac{1}{\sqrt{4 \pi(d-1) t}}(1+t)^{2 d-1}, \\
& M \sim(1-r) \frac{1}{\sqrt{4 \pi(d-1) t}}(1+t)^{2 d-1}+r(1+t)^{2 d-2} .
\end{aligned}
$$

Using the relations between $A_{1}, B_{1}$ and $M_{1}$ in (27), we obtain

$$
\begin{aligned}
A & \sim(d-1)^{2} r(t+1)^{2(d-2)}+\frac{(2 d-1)(t+1)^{2 d-2}}{8 t \sqrt{\pi} \sqrt{(d-1) t}}-\frac{(d-1)(t+1)^{2 d-1}}{16 t \sqrt{\pi}((d-1) t)^{3 / 2}} \\
& +\frac{1}{4}\left(\frac{(2 d-2)(2 d-1)(t+1)^{2 d-3}}{2 \sqrt{\pi} \sqrt{(d-1) t}}-\frac{(d-1)(2 d-1)(t+1)^{2 d-2}}{2 \sqrt{\pi}((d-1) t)^{3 / 2}}\right. \\
& \left.+\frac{3(d-1)^{2}(t+1)^{2 d-1}}{8 \sqrt{\pi}((d-1) t)^{5 / 2}}\right), \\
B & \sim(d-1) r(t+1)^{2 d-3}+\frac{1}{2}(1-r)\left(\frac{(2 d-1)(t+1)^{2 d-2}}{2 \sqrt{\pi} \sqrt{(d-1) t}}-\frac{(d-1)(t+1)^{2 d-1}}{4 \sqrt{\pi}((d-1) t)^{3 / 2}}\right) .
\end{aligned}
$$

Therefore, we get

$$
\begin{aligned}
f^{2} & =\frac{1}{\pi^{2}} \frac{A M-B^{2}}{M^{2}} \\
& \sim \frac{(1-r)(2(1-2 d)(r-1) t(t+1)+\sqrt{\pi} r(t(8 d+t-6)+1) \sqrt{(d-1) t})}{8 \pi^{2} t^{2}(t+1)((r-1)(t+1)-2 \sqrt{\pi} r \sqrt{(d-1) t})^{2}} .
\end{aligned}
$$

Denote the expression on the right-hand side by $f_{a}^{2}$. If $r=0$, we have

$$
f_{a}^{2}=\frac{2(2 d-1) t(t+1)}{8 \pi^{2} t^{2}(t+1)(t+1)^{2}}=\frac{2 d-1}{4 \pi^{2} t(t+1)^{2}},
$$

which means

$$
f_{a}=\frac{\sqrt{2 d-1}}{2 \pi \sqrt{t}(t+1)} .
$$

Therefore

$$
E \sim E_{a}:=2 \int_{0}^{1} f_{a} \mathrm{~d} t=2 \int_{0}^{1} \frac{\sqrt{2 d-1}}{2 \pi t^{1 / 2}(1+t)} \mathrm{d} t=\frac{\sqrt{2 d-1}}{2}=\mathcal{O}\left(d^{1 / 2}\right) .
$$

It remains to consider the case $0<r<1$. As the first asymptotic value of $E$ we compute

$$
E_{1}=2 \int_{0}^{1} f_{a}(t) \mathrm{d} t
$$

However, this formula is still not explicit since we need to take square root of $f_{a}$. Next we will offer another explicit approximation. To this end, we will further simplify $f_{a}$ asymptotically. Because

$$
(2(1-2 d)(r-1) t(t+1)+\sqrt{\pi} r(t(8 d+t-6)+1) \sqrt{(d-1) t}) \sim \sqrt{\pi} r t 8 d \sqrt{\mathrm{d} t}
$$

and

$$
((r-1)(t+1)-2 \sqrt{\pi} r \sqrt{(d-1) t})^{2} \sim 4 \pi r^{2} \mathrm{~d} t
$$


we obtain

$$
\begin{aligned}
f_{a}^{2} & =\frac{(1-r)(2(1-2 d)(r-1) t(t+1)+\sqrt{\pi} r(t(8 d+t-6)+1) \sqrt{(d-1) t})}{8 \pi^{2} t^{2}(t+1)((r-1)(t+1)-2 \sqrt{\pi} r \sqrt{(d-1) t})^{2}} \\
& \sim \frac{(1-r) \sqrt{\pi} r t 8 d \sqrt{\mathrm{d} t}}{8 \pi^{2} t^{2}(t+1) 4 \pi r^{2} \mathrm{~d} t}=\frac{\sqrt{d}(1-r)}{4 \pi^{5 / 2} r t^{3 / 2}(t+1)},
\end{aligned}
$$

which implies that

$$
f_{a} \sim \frac{d^{1 / 4}(1-r)^{1 / 2}}{2 \pi^{5 / 4} r^{1 / 2} t^{3 / 4}(t+1)^{1 / 2}}
$$

Hence, we obtain another approximation for $E(r, d)$ as follows.

$$
\begin{aligned}
E(r, d) \sim E_{2} & :=\int_{0}^{1} \frac{d^{1 / 4}(1-r)^{1 / 2}}{2 \pi^{5 / 4} r^{1 / 2} t^{3 / 4}(t+1)^{1 / 2}} \mathrm{~d} t \\
& =\frac{d^{1 / 4}(1-r)^{1 / 2}}{2 \pi^{5 / 4} r^{1 / 2}} \int_{0}^{1} \frac{1}{t^{3 / 4}(t+1)^{1 / 2}} \mathrm{~d} t \\
& =\frac{d^{1 / 4}(1-r)^{1 / 2}}{2 \pi^{5 / 4} r^{1 / 2}} \frac{8 \Gamma\left(\frac{5}{4}\right)^{2}}{\sqrt{\pi}} .
\end{aligned}
$$

The formal computations clearly show that the correlation $r$ between the coefficients $\{\beta\}$ significantly influences the expected number of equilibria $E(r, d)$ :

$$
E(r, d)= \begin{cases}\mathcal{O}\left(d^{1 / 2}\right), & \text { if } r=0, \\ \mathcal{O}\left(d^{1 / 4}\right), & \text { if } 0<r<1, \\ 0, & \text { if } r=1 .\end{cases}
$$

In Sect. 4.2 we will provide numerical verification for our formal computations.

Corollary 3 The expected number of stable equilibrium points $S E(r, d)$ follows the asymptotic behaviour

$$
S E(r, d)=\left\{\begin{array}{l}
\mathcal{O}\left(d^{1 / 2}\right), \quad \text { if } r=0, \\
\mathcal{O}\left(d^{1 / 4}\right), \quad \text { if } 0<r<1, \\
0, \quad \text { if } r=1 .
\end{array}\right.
$$

Proof This is a direct consequence of Theorems 3 and 1.

Remark 1 In "Appendix 6.4", we show the following asymptotic formula for $f(1 ; r, d)$

$$
f(1 ; r, d) \sim \frac{(d-1)^{1 / 4}(1-r)^{1 / 2}}{2 \sqrt{2} \pi^{5 / 4} r^{1 / 2}} .
$$

It is worth noticing that this asymptotic behaviour is of the same form as that of $E(r, d)$. 

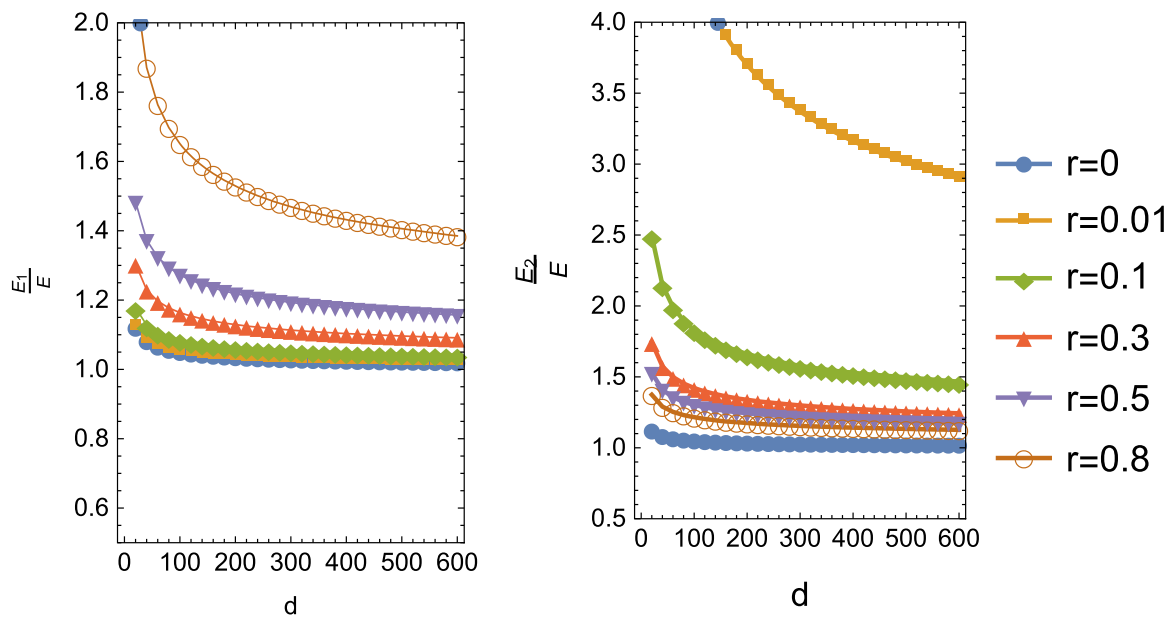

Fig. 2 Plot of $E_{1} / E(r, d)$ (left), and $E_{2} / E(r, d)$ (right). The figure shows that these ratios all converge to 1 when $d$ becomes large. We also notice that $E_{2}$ approximates $E$ better when $r$ is close to 1 while $E_{1}$ approximates $E$ better when $r$ is small

Table $1\left|\frac{E_{1}}{E}-1\right|$

\begin{tabular}{lllllll}
\hline$d$ & $r$ & 0.1 & 0.3 & 0.5 & 0.8 \\
\hline 20 & 0.119 & 0.126 & 0.178 & 0.305 & 0.484 & 1.106 \\
40 & 0.08 & 0.086 & 0.128 & 0.23 & 0.373 & 0.871 \\
120 & 0.045 & 0.049 & 0.08 & 0.154 & 0.257 & 0.616 \\
200 & 0.034 & 0.038 & 0.065 & 0.129 & 0.219 & 0.529 \\
320 & 0.027 & 0.03 & 0.055 & 0.111 & 0.19 & 0.461 \\
440 & 0.023 & 0.026 & 0.049 & 0.1 & 0.172 & 0.421 \\
600 & 0.019 & 0.023 & 0.044 & 0.091 & 0.157 & 0.385 \\
\hline
\end{tabular}

Table $2\left|\frac{E_{2}}{E}-1\right|$

\begin{tabular}{lllllll}
\hline$d$ & $r$ & & & & & \\
& 0 & 0.01 & 0.1 & 0.3 & 0.5 & 0.8 \\
\hline 20 & 0.119 & 5.855 & 1.495 & 0.745 & 0.528 & 0.374 \\
40 & 0.08 & 4.587 & 1.148 & 0.575 & 0.409 & 0.29 \\
120 & 0.045 & 3.186 & 0.782 & 0.397 & 0.285 & 0.203 \\
200 & 0.034 & 2.701 & 0.661 & 0.338 & 0.244 & 0.174 \\
320 & 0.027 & 2.322 & 0.568 & 0.293 & 0.212 & 0.152 \\
440 & 0.023 & 2.097 & 0.514 & 0.266 & 0.193 & 0.138 \\
600 & 0.019 & 1.9 & 0.467 & 0.243 & 0.176 & 0.127
\end{tabular}

\subsection{Asymptotic Behaviour of $E(r, d)$ : Numerical Investigation}

In this section, we numerically validate the asymptotic behaviour of $E(r, d)$ for large $d$ that is obtained in the previous section using formal analytical computations. In Fig. 2, Tables 1 and 2 we plot the ratios of the asymptotically approximations of $E(r, d)$ obtained in Sect. 4 
with itself, i.e. $E_{1} / E(r, d)$ and $E_{2} / E(r, d)$, for different values of $r$ and $d$. We observe that: for $r=0$ the approximation is good; while for $0<r<1$ : $E_{1}$ (respectively, $E_{2}$ ) approximates $E(r, d)$ better when $r$ is small (respectively, when $r$ is close to 1 ).

\section{Conclusion}

In this paper, we have studied the mean value, $E(r, d)$, of the number of internal equilibria in $d$-player two-strategy random evolutionary games where the entries of the payoff matrix are correlated random variables ( $r$ is the correlation). We have provided analytical formulas for $E(r, d)$ and proved that it is decreasing as a function of $r$. That is, our analysis has shown that decreasing the correlation among payoff entries leads to larger expected numbers of (stable) equilibrium points. This suggests that when payoffs obtained by a strategy for different group compositions are less correlated, it would lead to higher levels of strategic or behavioural diversity in a population. Thus, one might expect that when strategies behave conditionally on or even randomly for different group compositions, diversity would be promoted. Furthermore, we have shown that the asymptotic behaviour of $E(r, d)$ (and thus also of the mean number of stable equilibrium points, $S E(r, d)$ ), i.e. when the group size $d$ is sufficiently large, is highly sensitive to the correlation value $r$. Namely, $E(r, d)$ (and $S E(r, d)$ ) asymptotically behave in the order of $d^{1 / 2}$ for $r=0$ (i.e. the payoffs are independent for different group compositions), of $d^{1 / 4}$ for $0<r<1$ (i.e. non-extreme correlation), and 0 when $r=1$ (i.e. the payoffs are perfectly linear). It is also noteworthy that our numerical results showed that $E(r, d)$ increases with the group size $d$. In general, our findings might have important implications for the understanding of social and biological systems given the important roles of social and biological diversities, e.g. in the evolution of cooperative behaviour and population fitness distribution [37,47,60].

Moreover, we have explored further connections between EGT and random polynomial theory initiated in our previous works $[14,15]$. The random polynomial $P$ obtained from EGT (cf. (5)) differs from three well-known classes of random polynomials, namely Kac polynomials, elliptic polynomials and Weyl polynomials, that are investigated intensively in the literature. We elaborate further this difference in Sect. 6.6. In addition, as will be explained in Sect. 6.7, the set of positive roots of $P$ is the same as that of a Bernstein random polynomial. As a result, our work provides an analytical formula and asymptotic behaviour for the expected number of Bernstein random polynomials proving [18, Conjecture 4.7]. Thus, our work also contributes to the literature of random polynomial theory and to further its existing connection to EGT.

Although the expected number of internal equilibria provides macroscopic (average) information, to gain deeper insights into a multiplayer game such as possibilities of different states of biodiversity or the maintenance of biodiversity, it is crucial to analyse the probability distribution of the number of (stable) internal equilibria [27,37,61]. Thus a more subtle questions is: what is the probability, $p_{m}$, with $0 \leq m \leq d-1$, that a $d$-player two-strategy game attains $m$ internal equilibria? This question has been addressed for games with a small number of players [27,36]. We will tackle this more intricate question for arbitrary $d$ in a separate paper [17]. We expect that our work in this paper as well as in [17] will open up a new exciting avenue of research in the study of equilibrium properties of random evolutionary games. We discuss below some directions for future research.

Other types of correlations. In this paper we have assumed that the correlations $\operatorname{corr}\left(\beta_{i}, \beta_{j}\right)$ are constants for all pairs $i \neq j$. This is a fairly simple relation. Generally 
$\operatorname{corr}\left(\beta_{i}, \beta_{j}\right)$ may depend on $i$ and $j$ as showing in Lemma 1. Two interesting cases that are commonly studied in interacting particle systems are: (a) exponentially decay correlations, $\operatorname{corr}\left(\beta_{i}, \beta_{j}\right)=\rho^{|i-j|}$ for some $0<\rho<1$, and (b) algebraically decay correlations, $\operatorname{corr}\left(\beta_{i}, \beta_{j}\right)=(1+|i-j|)^{-\alpha}$ for some $\alpha>0$. These types of correlations have been studied in the literature for different types of random polynomials [13,22,53].

Universality phenomena. Recently, in [67] the authors proved, for other classes of random polynomials (such as Kac polynomials, Weyl polynomials and elliptic polynomials, see Sect. 6.6), an intriguing universal phenomenon: the asymptotic behaviour of the expected number of zeros in the non-gaussian case matches that of the gaussian case once one has performed appropriate normalizations. Further research is demanded to see whether this universality phenomenon holds true for the random polynomial (1).

Acknowledgements This paper was written partly when M. H. Duong was at the Mathematics Institute, University of Warwick, and was supported by ERC Starting Grant 335120. M. H. Duong and T. A. Han acknowledge Research in Pairs Grant (No. 41606) by the London Mathematical Society to support their collaborative research.

Open Access This article is distributed under the terms of the Creative Commons Attribution 4.0 International License (http://creativecommons.org/licenses/by/4.0/), which permits unrestricted use, distribution, and reproduction in any medium, provided you give appropriate credit to the original author(s) and the source, provide a link to the Creative Commons license, and indicate if changes were made.

\section{Appendix: Detailed Proofs and Computations}

This appendix consists of detailed proofs and computations of some lemmas and theorems in the main text.

\subsection{Proof of Lemma 1}

We have

$$
\begin{aligned}
\operatorname{cov}\left(\beta_{i}, \beta_{j}\right) & =\operatorname{cov}\left(a_{i}-b_{i}, a_{j}-b_{j}\right) \\
& =\operatorname{cov}\left(a_{i}, a_{j}\right)+\operatorname{cov}\left(b_{i}, b_{j}\right)-\operatorname{cov}\left(a_{i}, b_{j}\right)-\operatorname{cov}\left(b_{i}, a_{j}\right) \\
& =r_{a} \eta^{2}+r_{b} \eta^{2}-2 r_{a b} \eta^{2} \\
& =\left(r_{a}+r_{b}-2 r_{a b}\right) \eta^{2} .
\end{aligned}
$$

Similarly,

$$
\operatorname{var}\left(\beta_{i}\right)=\operatorname{var}\left(a_{i}-b_{i}\right)=\operatorname{cov}\left(a_{i}-b_{i}, a_{i}-b_{i}\right)=2 \eta^{2}-2 r_{a b}^{\prime} \eta^{2}=2\left(1-r_{a b}^{\prime}\right) \eta^{2} .
$$

Hence, the correlation between $\beta_{i}$ and $\beta_{j}$ is

$$
\operatorname{corr}\left(\beta_{i}, \beta_{j}\right)=\frac{\operatorname{cov}\left(\beta_{i}, \beta_{j}\right)}{\sqrt{\operatorname{var}\left(\beta_{i}\right) \operatorname{var}\left(\beta_{j}\right)}}=\frac{\left(r_{a}+r_{b}-2 r_{a b}\right) \eta^{2}}{2\left(1-r_{a b}^{\prime}\right) \eta^{2}}=\frac{r_{a}+r_{b}-2 r_{a b}}{2\left(1-r_{a b}^{\prime}\right)} .
$$

\subsection{Proof of Theorem 2-(1)}

We prove (14). We recall the following notations that have been used in the proof of Theorem 3. 


$$
\begin{aligned}
& M_{1}=M_{1}(t, d)=\sum_{i=0}^{d-1}\left(\begin{array}{c}
d-1 \\
i
\end{array}\right)^{2} t^{2 i}, \quad M_{2}=M_{2}(t, d)=(1+t)^{2(d-1)} \\
& A_{1}=A_{1}(t, d)=\sum_{i=0}^{d-1} i^{2}\left(\begin{array}{c}
d-1 \\
i
\end{array}\right)^{2} t^{2(i-1)}, \quad A_{2}=A_{2}(t, d)=(d-1)^{2}(1+t)^{2(d-2)} \\
& B_{1}=B_{1}(t, d)=\sum_{i=0}^{d-1} i\left(\begin{array}{c}
d-1 \\
i
\end{array}\right)^{2} t^{2 i-1}, \quad B_{2}=B_{2}(t, d)=(d-1)(1+t)^{2 d-3}, \\
& M=M(t ; r, d)=(1-r) M_{1}+r M_{2}, \quad A=A(t ; r, d)=(1-r) A_{1}+r A_{2}, \\
& B=B(t ; r, d)=(1-r) B_{1}+r B_{2} .
\end{aligned}
$$

Then the density function $f(t ; r, d)$ is expressed in terms of $M, A$ and $B$ as (for simplicity of notation we drop $r, d$ in $f$ in the following)

$$
f(t)=\frac{1}{\pi} \frac{\sqrt{A M-B^{2}}}{M} .
$$

Next, we compute $f(1 / t)$. According to [15], we have the following relations, where' denotes a derivative with respect to $t$,

$$
\begin{aligned}
& A_{1}(t)=\frac{1}{4 t}\left(t M_{1}^{\prime}(t)\right)^{\prime}=\frac{1}{4 t}\left(M_{1}^{\prime}(t)+t M_{1}^{\prime \prime}(t)\right), \quad B_{1}(t)=\frac{1}{2} M_{1}^{\prime}(t), \\
& M_{1}(1 / t)=t^{2-2 d} M_{1}(t), \\
& A_{1}(1 / t)=\frac{t}{4}\left[M_{1}^{\prime}(1 / t)+\frac{1}{t} M_{1}^{\prime \prime}(1 / t)\right] \\
& \quad=\frac{1}{4} t^{4-2 d}\left[4(d-1)^{2} M_{1}(t)+(5-4 d) t M_{1}^{\prime}(t)+t^{2} M_{1}^{\prime \prime}(t)\right], \\
& B_{1}(1 / t)=\frac{1}{2} M_{1}^{\prime}(1 / t)=-t^{3-2 d}\left[(1-d) M_{1}(t)+\frac{1}{2} t M_{1}^{\prime}(t)\right] .
\end{aligned}
$$

Using the relations between $A_{1}, B_{1}$ and $M_{1}$ in (27), we transform further $A_{1}(1 / t)$ and $B_{1}(1 / t)$

$$
\begin{aligned}
A_{1}(1 / t) & =\frac{1}{4} t^{4-2 d}\left[4(d-1)^{2} M_{1}(t)+4(1-d) t M_{1}^{\prime}(t)+t\left(M_{1}^{\prime}(t)+t M_{1}^{\prime \prime}(t)\right)\right] \\
& =t^{4-2 d}\left[4(d-1)^{2} M_{1}(t)+4(1-d) t M_{1}^{\prime}(t)+t^{2} A_{1}(t)\right], \\
B_{1}(1 / t) & =-t^{3-2 d}\left[(1-d) M_{1}(t)+\frac{1}{2} t M_{1}^{\prime}(t)\right]=t^{2-2 d}\left[(d-1) M_{1}(t)-t B_{1}(t)\right] .
\end{aligned}
$$

Using explicit formulas of $M_{2}, A_{2}$ and $B_{2}$, we get

$$
M_{2}(1 / t)=t^{2-2 d} M_{2}(t), \quad A_{2}(1 / t)=t^{4-2 d} A_{2}(t), \quad B_{2}(1 / t)=t^{3-2 d} B_{2}(t) .
$$

Therefore, we obtain

$$
\begin{aligned}
M(1 / t)= & (1-r) M_{1}(1 / t)+r M_{2}(1 / t) \\
& =t^{2-2 d}\left[(1-r) M_{1}(t)+r M_{2}(t)\right]=t^{2-2 d} M(t), \\
A(1 / t)= & t^{4-4 d}\left[(1-r)\left((d-1)^{2} M_{1}(t)+(1-d) t M_{1}^{\prime}(t)+t^{2} A_{1}(t)\right)+r A_{2}(t)\right], \\
B(1 / t)= & t^{3-2 d}\left[(1-r)\left((d-1) M_{1}(t)-t B_{1}(t)\right)+r B_{2}(t)\right],
\end{aligned}
$$




$$
\begin{aligned}
M(1 / t) A(1 / t)= & t^{6-4 d}\left[(1-r)^{2}\left((d-1)^{2} M_{1}(t)+(1-d) t M_{1}^{\prime}(t)+t^{2} A_{1}(t)\right) M_{1}(t)\right. \\
& +r(1-r)\left(\left((d-1)^{2} M_{1}(t)+(1-d) t M_{1}^{\prime}(t)+t^{2} A_{1}(t)\right) M_{2}(t)\right. \\
& \left.\left.+A_{2}(t) M_{1}(t)\right)+r^{2} A_{2}(t) M_{2}(t)\right] \\
B(1 / t)^{2}= & t^{6-4 d}\left[(1-r)^{2}\left((d-1) M_{1}(t)-t B_{1}(t)\right)^{2}\right. \\
& \left.+2 r(1-r)\left((d-1) M_{1}(t)-t B_{1}(t)\right) B_{2}(t)+r^{2} B_{2}(t)^{2}\right]
\end{aligned}
$$

So we have

$$
\begin{aligned}
& M(1 / t) A(1 / t)-B(1 / t)^{2} \\
&= t^{6-4 d}\left[(1-r)^{2}\left((1-d) t M_{1}(t) M_{1}^{\prime}(t)+t^{2} A_{1}(t) M_{1}(t)+2(d-1) M_{1}(t) B_{1}(t)\right)\right. \\
&-t^{2} B_{1}(t)^{2}+r(1-r)\left((d-1)^{2} M_{1}(t) M_{2}(t)+(1-d) t M_{1}^{\prime}(t) M_{2}(t)\right. \\
&\left.+t^{2} A_{1}(t) M_{2}(t)+A_{2}(t) M_{1}(t)-2\left((d-1) M_{1}(t)-t B_{1}(t)\right) B_{2}(t)\right) \\
&\left.+r^{2}\left(A_{2}(t) M_{2}(t)-B_{2}(t)^{2}\right)\right] .
\end{aligned}
$$

Using the relations (27) and explicit formulas of $A_{2}, B_{2}, M_{2}$ we get

$$
\begin{aligned}
& A_{2}(t) M_{2}(t)-B_{2}^{2}(t)=0, \\
& (1-d) t M_{1}(t) M_{1}^{\prime}(t)+2(d-1) M_{1}(t) B_{1}(t)=(d-1) M_{1}(t)\left[2 B_{1}(t)-M_{1}^{\prime}(t)\right]=0, \\
& (d-1)^{2} M_{1}(t) M_{2}(t)+A_{2}(t) M_{1}(t)-2(d-1) M_{1}(t) B_{2}(t) \\
& \quad=M_{1}(t)\left((d-1) M_{2}(t)+A_{2}(t)-2(d-1) B_{2}(t)\right) \\
& \quad=t^{2} M_{1}(t) A_{2}(t), \\
& (1-d) t M_{1}^{\prime}(t) M_{2}(t)+2 t B_{1}(t) B_{2}(t)=2(1-d) t B_{1}(t) M_{2}(t)+2 t B_{1}(t) B_{2}(t) \\
& \quad=B_{1}(t)\left(2(1-d) t M_{2}(t)+2 t B_{2}(t)\right) \\
& \quad=-2 t^{2} B_{1}(t) B_{2}(t) .
\end{aligned}
$$

Substituting these computations into (29), we obtain

$$
\begin{aligned}
M & (1 / t) A(1 / t)-B(1 / t)^{2} \\
= & t^{8-4 d}\left[(1-r)^{2}\left(A_{1}(t) M_{1}(t)-B_{1}(t)^{2}\right)+r(1-r)\left(M_{1}(t) A_{2}(t)\right.\right. \\
& \left.\left.+M_{2}(t) A_{1}(t)-2 B_{1}(t) B_{2}(t)\right)\right] \\
& =t^{8-4 d}\left[\left((1-r) M_{1}(t)+r M_{2}(t)\right)\left((1-r) A_{1}(t)+r A_{2}(t)\right)\right.
\end{aligned}
$$




$$
\begin{aligned}
& \left.-\left((1-r) B_{1}(t)+r B_{2}(t)\right)^{2}\right] \\
= & t^{8-4 d}\left(M(t) A(t)-B(t)^{2}\right) .
\end{aligned}
$$

Finally, we get

$$
\begin{aligned}
f(1 / t) & =\frac{1}{\pi} \frac{\sqrt{A(1 / t) M(1 / t)-B(1 / t)^{2}}}{M(1 / t)}=\frac{1}{\pi} \frac{t^{4-2 d} \sqrt{A(t) M(t)-B^{2}(t)}}{t^{2-2 d} M(t)} \\
& =\frac{1}{\pi} t^{2} \frac{\sqrt{A(t) M(t)-B^{2}(t)}}{M(t)}=t^{2} f(t) .
\end{aligned}
$$

\subsection{Proof of Lemma 3}

The probability distribution, $f_{Z}$, of $Z=X-Y$ can be found via the joint probability distribution $f_{X, Y}$ as

$$
f_{Z}(z)=\int_{-\infty}^{\infty} f_{X, Y}(x, x-z) d x=\int_{-\infty}^{\infty} f_{X, Y}(y+z, y) d y .
$$

Therefore, using the symmetry of $f_{X, Y}$ we get

$$
f_{Z}(-z)=\int_{-\infty}^{\infty} f_{X, Y}(x, x+z) d x=\int_{-\infty}^{\infty} f_{X, Y}(x+z, x) d x=f_{Z}(z) .
$$

If $X$ and $Y$ are iid with the common probability distribution $f$, then

$$
f_{X, Y}(x, y)=f(x) f(y),
$$

which is symmetric with respect to $x$ and $y$, i.e. $X$ and $Y$ are exchangeable.

\subsection{Computations of $f(1 ; r, d)$}

Substituting $t=1$ into expressions of $A, B, M$ at the beginning of the proof of Theorem 2, we obtain

$$
\begin{aligned}
M(1 ; r, d) & =(1-r) \sum_{k=0}^{d-1}\left(\begin{array}{c}
d-1 \\
k
\end{array}\right)^{2}+r 2^{2(d-1)}=(1-r)\left(\begin{array}{c}
2(d-1) \\
d-1
\end{array}\right)+r 2^{2(d-1)}, \\
A(1 ; r, d) & =(1-r)(d-1)^{2} M(1 ; r, d-1)+r(d-1)^{2} 2^{2(d-2)} \\
& =(1-r)(d-1)^{2}\left(\begin{array}{c}
2(d-2) \\
d-2
\end{array}\right)+r(d-1)^{2} 2^{2(d-2)} \\
B(1 ; r, d) & =(1-r) \sum_{k=1}^{d-1} k\left(\begin{array}{c}
d-1 \\
k
\end{array}\right)^{2}+r(d-1) 2^{2 d-3)} \\
& =(1-r) \frac{d-1}{2}\left(\begin{array}{c}
2(d-1) \\
d-1
\end{array}\right)+r(d-1) 2^{2 d-3}
\end{aligned}
$$


Therefore,

$$
\begin{aligned}
A M-B^{2}= & (1-r)^{2}(d-1)^{2}\left(\begin{array}{c}
2(d-1) \\
d-1
\end{array}\right)\left[\left(\begin{array}{c}
2(d-2) \\
d-2
\end{array}\right)-\frac{1}{4}\left(\begin{array}{c}
2(d-1) \\
d-1
\end{array}\right)\right] \\
& +r(1-r)(d-1)^{2} 2^{2(d-2)}\left[4\left(\begin{array}{c}
2(d-2) \\
d-2
\end{array}\right)+\left(\begin{array}{c}
2(d-1) \\
d-1
\end{array}\right)-2\left(\begin{array}{c}
2(d-1) \\
d-1
\end{array}\right)\right] \\
= & (1-r)^{2}(d-1)^{2}\left(\begin{array}{c}
2(d-1) \\
d-1
\end{array}\right)\left[\left(\begin{array}{c}
2(d-2) \\
d-2
\end{array}\right)-\frac{1}{4}\left(\begin{array}{c}
2(d-1) \\
d-1
\end{array}\right)\right] \\
& +r(1-r)(d-1)^{2} 2^{2(d-1)}\left[\left(\begin{array}{c}
2(d-2) \\
d-2
\end{array}\right)-\frac{1}{4}\left(\begin{array}{c}
2(d-1) \\
d-1
\end{array}\right)\right] \\
= & (1-r)(d-1)^{2}\left[\left(\begin{array}{c}
2(d-2) \\
d-2
\end{array}\right)-\frac{1}{4}\left(\begin{array}{c}
2(d-1) \\
d-1
\end{array}\right)\right] \\
& {\left[(1-r)\left(\begin{array}{c}
2(d-1) \\
d-1
\end{array}\right)+r 2^{2 d-1}\right] . }
\end{aligned}
$$

Substituting this expression and that of $M$ into (26), we get

$$
\begin{aligned}
f(1 ; r, d) & =\frac{1}{\pi} \frac{\sqrt{A M-B^{2}}}{M} \\
& =\frac{1}{\pi}(d-1) \sqrt{1-r} \times \sqrt{\frac{\left(\begin{array}{c}
2(d-2) \\
d-2
\end{array}\right)-\frac{1}{4}\left(\begin{array}{c}
2(d-1) \\
d-1
\end{array}\right)}{(1-r)\left(\begin{array}{c}
2(d-1) \\
d-1
\end{array}\right)+r 2^{2(d-1)}}} \\
& =\frac{1}{\pi}(d-1) \sqrt{1-r} \times \sqrt{\frac{\left(\begin{array}{c}
2(d-1) \\
d-1
\end{array}\right) \frac{1}{4(2 d-3)}}{(1-r)\left(\begin{array}{c}
2(d-1) \\
d-1
\end{array}\right)+r 2^{2(d-1)}}} \\
& =\frac{1}{\pi} \frac{d-1}{2 \sqrt{2 d-3}} \sqrt{\frac{(1-r)\left(\begin{array}{c}
2(d-1) \\
d-1
\end{array}\right)}{(1-r)\left(\begin{array}{c}
2(d-1) \\
d-1
\end{array}\right)+r 2^{2(d-1)}} .}
\end{aligned}
$$

If $r=1$ then $f(1 ; r, d)=0$. If $r<1$ then

$$
f(1 ; r, d)=\frac{1}{\pi} \frac{d-1}{2 \sqrt{2 d-3}} \sqrt{\frac{1}{1+\alpha}} \text { where } \alpha=\frac{r}{1-r} \frac{2^{2(d-1)}}{\left(\begin{array}{c}
2(d-1) \\
d-1
\end{array}\right)} .
$$

By Stirling formula, we have

$$
\left(\begin{array}{c}
2 n \\
n
\end{array}\right) \sim \frac{4^{n}}{\sqrt{\pi n}} \text { for large } n .
$$

It implies that for $0<r<1$ and for large $d, \alpha \sim \frac{r}{1-r} \sqrt{\pi(d-1)}$, from which we obtain

$$
f(1 ; r, d) \sim \frac{1}{\pi} \frac{d-1}{2 \sqrt{2 d-3}} \sqrt{\frac{1}{1+\frac{r}{1-r} \sqrt{\pi(d-1)}}} \sim \frac{(d-1)^{1 / 4}(1-r)^{1 / 2}}{2 \sqrt{2} \pi^{5 / 4} r^{1 / 2}} .
$$




\subsection{Some Technical Lemmas Used in Proof of Theorem 3}

We need the following proposition.

Proposition 2 The following inequality holds

$$
2(t+1) B\left[B_{1}(1+t)-M_{1}(d-1)\right]<M\left[A_{1}(1+t)^{2}-M_{1}(d-1)^{2}\right] .
$$

To prove Proposition 2, we need several auxiliary lemmas. We note that throughout this section

$$
x=\frac{1+t^{2}}{1-t^{2}}, \quad 0<t<1,
$$

and $P_{d}(z)$ is the Legendre polynomial of degree $d$ which is defined through the following recurrent relation

$$
(2 d+1) z P_{d}(z)=(d+1) P_{d+1}(z)+d P_{d-1}(z) ; \quad P_{0}(z)=1, \quad P_{1}(z)=z .
$$

We refer to [15] for more information on the Legendre polynomial and its connections to evolutionary game theory.

Lemma 4 It holds that

$$
\lim _{d \rightarrow \infty} \frac{P_{d}(x)}{P_{d+1}(x)}=x-\sqrt{x^{2}-1} .
$$

Note that $x=\frac{1+t^{2}}{1-t^{2}}$, we can write the above limit as

$$
\lim _{d \rightarrow \infty} \frac{P_{d}(x)}{P_{d+1}(x)}=\frac{1-t}{1+t} .
$$

Proof According to [15, Lemma 4], we have

$$
P_{d}(x)^{2} \leq P_{d+1}(x) P_{d-1}(x) .
$$

Since $P_{d}(x)>0$, we get

$$
x \geq \frac{1}{x}=\frac{P_{0}(x)}{P_{1}(x)} \geq \frac{P_{1}(x)}{P_{2}(x)} \geq \cdots \geq \frac{P_{d-1}(x)}{P_{d}(x)} \geq \frac{P_{d}(x)}{P_{d+1}(x)} \geq 0 .
$$

Therefore, there exists a function $0 \leq f(x) \leq \frac{1}{x}$ such that

$$
\lim _{d \rightarrow \infty} \frac{P_{d}(x)}{P_{d+1}(x)}=f(x) .
$$

From the recursive relation (31), we have

$$
(2 d+1) x=(d+1) \frac{P_{d+1}(x)}{P_{d}(x)}+d \frac{P_{d-1}(x)}{P_{d}(x)},
$$

which implies that

$$
\frac{d+1}{d}=\frac{\frac{P_{d-1}(x)}{P_{d}(x)}-x}{x-\frac{P_{d+1}(x)}{P_{d}(x)}} .
$$


Taking the limit $d \rightarrow \infty$ both sides, we obtain

$$
1=\frac{f(x)-x}{x-\frac{1}{f(x)}} .
$$

Solving this equation for $f(x)$, requiring that $0 \leq f(x) \leq \frac{1}{x} \leq x$ we obtain $f(x)=$ $x-\sqrt{x^{2}-1}$.

Lemma 5 The following inequalities hold

$$
(1-t)^{2} \leq\left(1-t^{2}\right) \frac{P_{d}(x)}{P_{d+1}(x)} \leq 1+t^{2} .
$$

Proof By dividing by $1-t^{2}$, the required inequalities are equivalent to (recalling that $0<$ $t<1$ )

$$
\frac{1-t}{1+t} \leq \frac{P_{d}(x)}{P_{d+1}(x)} \leq x,
$$

which are true following from (32) and (33).

Lemma 6 The following equality holds

$$
2(d-1) t\left[B_{1}(1+t)-M_{1}(d-1)\right]=(t-1)\left[A_{1}(1+t)^{2}-M_{1}(d-1)^{2}\right] .
$$

Proof The stated equality is simplified to

$$
A_{1}\left(t^{2}-1\right)+M_{1}(d-1)^{2}-2(d-1) t B_{1}=0 .
$$

We use the following results from [15, Lemma $3 \&$ Section 6.2]

$$
\begin{aligned}
A_{1}(t, d)= & (d-1)^{2} M_{1}(t, d-1)=(d-1)^{2}\left(1-t^{2}\right)^{d-2} P_{d-2}(x), \\
M_{1}= & \left(1-t^{2}\right)^{d-1} P_{d-1}(x), \\
B_{1}= & \frac{M_{1}^{\prime}}{2} \\
= & M_{1}\left(\frac{-t(d-1)}{1-t^{2}}+\frac{2 t}{\left(1-t^{2}\right)^{2}} \frac{P_{d-1}^{\prime}}{P_{d-1}}(x)\right) \\
= & M_{1}\left(\frac{-t(d-1)}{1-t^{2}}+\frac{2 t}{\left(1-t^{2}\right)^{2}} \frac{(d-1)\left(1-t^{2}\right)^{2}}{4 t^{2}}\left(\frac{1+t^{2}}{1-t^{2}}-\frac{P_{d-2}(x)}{P_{d-1}(x)}\right)\right) \\
= & M_{1}\left(\frac{-t(d-1)}{1-t^{2}}+\frac{d-1}{2 t}\left(\frac{1+t^{2}}{1-t^{2}}-\frac{P_{d-2}(x)}{P_{d-1}(x)}\right)\right) \\
= & (d-1)\left(-t\left(1-t^{2}\right)^{d-2} P_{d-1}(x)\right. \\
& \left.+\frac{\left(1+t^{2}\right)\left(1-t^{2}\right)^{d-2} P_{d-1}(x)-\left(1-t^{2}\right)^{d-1} P_{d-2}(x)}{2 t}\right) .
\end{aligned}
$$

Substituting these expressions into the left-hand side of (36), we obtain 0 as required.

Lemma 7 The following inequality holds

$$
\begin{aligned}
& (t-1)\left[B_{1}(1+t)-M_{1}(d-1)\right] \geq 0, \\
& \left(t^{2}-1\right) B_{1}-(d-1) t M_{1} \leq 0, \\
& \left(t^{2}-1\right)\left(B_{2}-B_{1}\right)-(d-1) t\left(M_{2}-M_{1}\right) \leq 0 .
\end{aligned}
$$


Proof We prove (39) first. Since $M_{1}>0,(39)$ is simplified to

$$
\left(t^{2}-1\right) \frac{B_{1}}{M_{1}}-(d-1)(t-1) \geq 0 .
$$

Using the relation (38) between $B_{1}$ and $M_{1}$, we obtain

$$
\begin{aligned}
& \left(t^{2}-1\right) \frac{B_{1}}{M_{1}}-(d-1)(t-1) \\
& \quad=\left(t^{2}-1\right) \frac{M_{1}^{\prime}}{2 M_{1}}-(d-1)(t-1) \\
& \quad=\left(t^{2}-1\right)\left[\frac{-t(d-1)}{1-t^{2}}+\frac{2 t}{\left(1-t^{2}\right)^{2}} \frac{P_{d-1}^{\prime}}{P_{d-1}}\left(\frac{1+t^{2}}{1-t^{2}}\right)\right]-(d-1)(t-1) \\
& \quad=(d-1)+\frac{2 t}{t^{2}-1} \frac{P_{d-1}^{\prime}}{P_{d-1}}(x) .
\end{aligned}
$$

Now using the following relation [15, Eq. (49)]

$$
\frac{P_{d-1}^{\prime}(x)}{P_{d-1}(x)}=\frac{d-1}{x^{2}-1}\left(x-\frac{P_{d-2}(x)}{P_{d-1}(x)}\right)=\frac{(d-1)\left(1-t^{2}\right)^{2}}{4 t^{2}}\left(\frac{1+t^{2}}{1-t^{2}}-\frac{P_{d-2}(x)}{P_{d-1}(x)}\right),
$$

we obtain

$$
\begin{aligned}
\left(t^{2}-1\right) \frac{B_{1}}{M_{1}}-(d-1)(t-1) & =(d-1)\left(1-\frac{1+t^{2}}{2 t}-\frac{t^{2}-1}{2 t} \frac{P_{d-2}(x)}{P_{d-1}(x)}\right) \\
& =-\frac{d-1}{2 t}\left[(1-t)^{2}-\left(1-t^{2}\right) \frac{P_{d-1}}{P_{d-1}}(x)\right] \\
& \geq 0,
\end{aligned}
$$

where the last inequality follows from Lemma 5. This establishes (39).

Next, we prove (40), which can be simplified to

$$
(d-1)\left(-\frac{1+t^{2}}{2 t}-\frac{t^{2}-1}{2 t} \frac{P_{d-2}(x)}{P_{d-1}(x)}\right) \leq 0,
$$

which is in turn equivalent to

$$
\left(1-t^{2}\right) \frac{P_{d-2}(x)}{P_{d-1}(x)} \leq 1+t^{2} .
$$

This has been proved in Lemma 5 .

Finally, we prove (41). First, we simplify

$$
\begin{aligned}
\left(t^{2}-1\right) B_{2}-(d-1) t M_{2} & =(d-1)\left(t^{2}-1\right)(1+t)^{2 d-3}-(d-1) t(1+t)^{2 d-2} \\
& =-(d-1)(1+t)^{2 d-2} .
\end{aligned}
$$

Thus, (41) is equivalent to

$$
(d-1)\left(\frac{1+t^{2}}{2 t}+\frac{t^{2}-1}{2 t} \frac{P_{d-2}(x)}{P_{d-1}(x)}-(1+t)^{2 d-2}\right) \leq 0 .
$$

This clearly holds because $t \geq 0$ and from the proof of the first inequality we already know that

$$
\frac{1+t^{2}}{2 t}+\frac{t^{2}-1}{2 t} \frac{P_{d-2}(x)}{P_{d-1}(x)}-1 \leq 0 .
$$


Thus, we finish the proof of the lemma.

We are now ready to provide a proof of Proposition 2.

Proof (Proof of Proposition 2) From Lemma 6, since $M_{1}, A_{1}$ and $B_{1}$ are polynomials (of $t$ ) with integer coefficients, there exists a polynomial $S(t)$ such that

$B_{1}(1+t)-M_{1}(d-1)=(t-1) S(t)$ and $A_{1}(1+t)^{2}-M_{1}(d-1)^{2}=2(d-1) t S(t)$

If follows from (39) that $S(t) \geq 0$. Next, we will prove that

$$
\begin{aligned}
& 2(t+1) B_{1}\left[B_{1}(1+t)-M_{1}(d-1)\right] \leq M_{1}\left[A_{1}(1+t)^{2}-M_{1}(d-1)^{2}\right], \\
& 2(t+1)\left(B_{2}-B_{1}\right)\left[B_{1}(1+t)-M_{1}(d-1)\right] \leq\left(M_{2}-M_{1}\right)\left[A_{1}(1+t)^{2}-M_{1}(d-1)^{2}\right] .
\end{aligned}
$$

Indeed, these inequalities can be rewritten as

$$
\begin{aligned}
& 2 S(t)\left[\left(t^{2}-1\right) b_{1}-(d-1) t m_{1}\right]<0, \\
& 2 S(t)\left[\left(t^{2}-1\right)\left(b_{2}-b_{1}\right)-(d-1) t\left(m_{2}-m_{1}\right)\right]<0,
\end{aligned}
$$

which hold due to Lemma 7. Multiplying (44) with $r>0$ and adding with (43) yield the assertion of Proposition 2.

\subsection{Comparison with Known Results for Other Classes of Random Polynomials}

The distribution and expected number of real zeros of a random polynomial has been a topic of intensive research dating back to 1932 with Block and Pólya [8], see for instance the monograph [13] for a nice exposition and [44,67] for recent results and discussions. The most general form of a random polynomial is given by

$$
\mathcal{P}_{d}(z)=\sum_{i=0}^{d} c_{i} \xi_{i} z^{i},
$$

where $c_{i}$ are deterministic coefficients which may depend on both $d$ and $i$, and $\xi_{i}$ are random variables. The most three well-known classes of polynomials are

(i) Kac polynomials: $c_{i}:=1$,

(ii) Weyl (or flat) polynomials: $c_{i}:=\frac{1}{i !}$,

(iii) Elliptic (or binomial) polynomials: $c_{i}:=\sqrt{\left(\begin{array}{l}d \\ i\end{array}\right)}$.

The expected number of real zeros of these polynomials when $\left\{\xi_{i}\right\}$ are i.i.d standard normal variables is, respectively, $E_{K} \sim \frac{2}{\pi} \log d, E_{W} \sim \frac{2}{\pi} \sqrt{d}$ and $E_{E}=\sqrt{d}$, see, e.g. [67] and references therein. Random polynomials in which $\xi_{i}$ are correlated random variables have also attracted considerable attention, see, e.g. [13,21-23,51-54] and references therein. Particularly, when $\left\{\xi_{i}\right\}$ satisfy the same assumption as in this paper, it has been shown, in [51] for the Kac polynomial that $E_{K} \sim \frac{2}{\pi} \sqrt{1-r^{2}} \log d$, and in [23] for elliptic polynomials that $E_{E} \sim \frac{\sqrt{d}}{2}$.

The random polynomial $P$ arising from evolutionary game theory in this paper, see Equation (1), corresponds to $c_{i}=\left(\begin{array}{c}d-1 \\ i\end{array}\right)$; thus it differs from all the above three classes. In 
Sect. 6.7, we show that a root of $P$ is also a root of the Bernstein polynomial. Therefore, we also obtain an asymptotic formula for the expected number of real zeros of the random Bernstein polynomial. We anticipate that evolutionary game theory and random polynomial theory have deeply undiscovered connections in different scenarios. We shall continue this development in a forthcoming paper.

\subsection{On the Expected Number of Real Zeros of a Random Bernstein Polynomial of Degree $d$}

Similarly as in [15, Corollary 2], as a by-product of Theorem 1, we obtain an asymptotic formula for the expected number of real zeros, $E_{\mathcal{B}}$, of a random Bernstein polynomial of degree $d$

$$
\mathcal{B}(x)=\sum_{k=0}^{d} \beta_{k}\left(\begin{array}{l}
d \\
k
\end{array}\right) x^{k}(1-x)^{d-k},
$$

where $\beta_{k}$ are i.i.d. standard normal distributions. Indeed, by changing of variables $y=\frac{x}{1-x}$ as in Sect. 2, zeros of $\mathcal{B}(x)$ are the same as those of the following random polynomial

$$
\tilde{\mathcal{B}}(y)=\sum_{k=0}^{d} \beta_{k}\left(\begin{array}{l}
d \\
k
\end{array}\right) y^{k} .
$$

As a consequence of Theorem 1 , the expected number of real zeros, $E_{\mathcal{B}}$, of a random Bernstein polynomial of degree $d$ is given by

$$
E_{\mathcal{B}}=2 E(0, d+1) \sim \sqrt{2 d+1} .
$$

This proves Conjecture 4.7 in [18]. Connections between EGT and Bernstein polynomials have also been discussed in [48].

\section{References}

1. Abel NH (1824) Mémoire sur les équations algébriques, où l'on démontre l'impossibilité de la résolution de l'équation générale du cinquiéme degré. Abel's Ouvres 1:28-33

2. Altenberg L (2010) Proof of the feldman-karlin conjecture on the maximum number of equilibria in an evolutionary system. Theor Popul Biol 77(4):263-269

3. Axelrod R (1984) The evolution of cooperation. Basic Books, New York

4. Broom M, Cannings C, Vickers GT (1997) Multi-player matrix games. Bull Math Biol 59(5):931-952

5. Berg J, Engel A (1998) Matrix games, mixed strategies, and statistical mechanics. Phys Rev Lett 81:4999_ 5002

6. Bach LA, Helvik T, Christiansen FB (2006) The evolution of n-player cooperation threshold games and ess bifurcations. J Theor Biol 238(2):426-434

7. Bender CM, Orszag SA (1999) Advanced mathematical methods for scientists and engineers: I: asymptotic methods and perturbation theory. Springer, New York

8. Bloch A, Pólya G (1932) On the roots of certain algebraic equations. Proc Lond Math Soc S2-33(1):102

9. Broom M, Rychtár J (2013) Game-theoretical models in biology. CRC Press, Boca Raton

10. Broom M, Rychtáŕ J (2016) Nonlinear and multiplayer evolutionary games. Springer International Publishing, Cham, pp 95-115

11. Broom M (2000) Bounds on the number of esss of a matrix game. Math Biosci 167(2):163-175

12. Broom M (2003) The use of multiplayer game theory in the modeling of biological populations. Comm Theor Biol 8:103-123

13. Bharucha-Reid AT, Sambandham M (1986) Random polynomials. Probability and mathematical statistics. Academic Press Inc, Orlando 
14. Duong MH, Han TA (2015) On the expected number of equilibria in a multi-player multi-strategy evolutionary game. Dyn Games Appl, pp 1-23

15. Duong MH, Han TA (2016) Analysis of the expected density of internal equilibria in random evolutionary multi-player multi-strategy games. J Math Biol 73(6):1727-1760

16. Dobramysl U, Mobilia M, Pleimling M, Täuber UC (2018) Stochastic population dynamics in spatially extended predator-prey systems. J Phys A: Math Theor 51(6):063001

17. Duong MH, Tran HM, Han TA (2017) On the distribution of the number of internal equilibria in random evolutionary games. J Math Biol. https://doi.org/10.1007/s00285-018-1276-0 (in press)

18. Emiris IZ, Galligo A, Tsigaridas EP (2010) Random polynomials and expected complexity of bisection methods for real solving. In: Proceedings of the 2010 international symposium on symbolic and algebraic computation, ISSAC '10, ACM, New York, pp 235-242

19. Edelman A, Kostlan E (1995) How many zeros of a random polynomial are real? Bull Amer Math Soc 32(1):1-37

20. Fudenberg D, Harris C (1992) Evolutionary dynamics with aggregate shocks. J Econ Theory 57(2):420441

21. Farahmand K, Nezakati A (2005) Algebraic polynomials with dependent random coeffcients. Commun Appl Anal 9:94-104 01/2005

22. Farahmand K, Nezakati A (2010) Real zeros of algebraic polynomials with dependent random coefficients. Stoch Anal Appl 28(3):558-564

23. Farahmand K, Nezakati A (2011) The expected number of real zeros of algebraic polynomials with dependent and non-identical random coefficients. Stoch Anal Appl 29(3):452-456

24. Friedman D (1998) On economic applications of evolutionary game theory. J Evol Econ 8(1):15-43

25. Galla T, Farmer JD (2013) Complex dynamics in learning complicated games. Proc Natl Acad Sci 110(4):1232-1236

26. Gross T, Rudolf L, Levin SA, Dieckmann U (2009) Generalized models reveal stabilizing factors in food webs. Science 325(5941):747-750

27. Gokhale CS, Traulsen A (2010) Evolutionary games in the multiverse. Proc Natl Acad Sci USA 107(12):5500-5504

28. Gokhale CS, Traulsen A (2014) Evolutionary multiplayer games. Dyn Games Appl 4(4):468-488

29. Han TA (2013) Intention recognition, commitments and their roles in the evolution of cooperation: from artificial intelligence techniques to evolutionary game theory models, vol 9. Springer SAPERE series

30. Hardin G (1968) The tragedy of the commons. Science 162:1243-1248

31. Hauert C, De Monte S, Hofbauer J, Sigmund K (2002) Replicator dynamics for optional public good games. J Theor Biol 218:187-194

32. Hauert C, Michor F, Nowak MA, Doebeli M (2006) Synergy and discounting of cooperation in social dilemmas. J Theor Biol 239:195-202

33. Han TA, Pereira LM, Lenaerts T (2015) Avoiding or restricting defectors in public goods games? J R Soc Interface 12(103):20141203

34. Han TA, Pereira LM, Lenaerts T (2017) Evolution of commitment and level of participation in public goods games. Auton Agent Multi-Agent Syst 31(3):561-583

35. Hofbauer J, Sigmund K (1998) Evolutionary games and population dynamics. Cambridge University Press, Cambridge

36. Han TA, Traulsen A, Gokhale CS (2012) On equilibrium properties of evolutionary multi-player games with random payoff matrices. Theor Popul Biol 81(4):264-272

37. Levin SA (2000) Multiple scales and the maintenance of biodiversity. Ecosystems 3(6):498-506

38. Levin Simon A (2000) Multiple scales and the maintenance of biodiversity. Ecosystems 3:498-506

39. May RM (2001) Stability and complexity in model ecosystems, vol 6. Princeton University Press, Princeton

40. Maynard Smith J (1982) Evolution and the theory of games. Cambridge University Press, Cambridge

41. Smith J Maynard, Price GR (1973) The logic of animal conflict. Nature 246:15-18

42. Nash JF (1950) Equilibrium points in n-person games. Proc Natl Acad Sci USA 36:48-49

43. Nowak AS, Collins KR (2000) Reliability of structures. McGraw-Hill, McGraw-Hill civil engineering series

44. Nguyen H, Nguyen $\mathrm{O}, \mathrm{Vu} \mathrm{V}$ (2016) On the number of real roots of random polynomials. Commun Contemp Math 18(04):1550052

45. Nowak MA (2006) Evolutionary dynamics. Harvard University Press, Cambridge, MA

46. Pennisi E (2005) How did cooperative behavior evolve? Science 309(5731):93-93

47. Peña J (2012) Group-size diversity in public goods games. Evolution 66(3):623-636

48. Peña J, Lehmann L, Nöldeke G (2014) Gains from switching and evolutionary stability in multi-player matrix games. J Theor Biol 346:23-33 
49. Perc M, Szolnoki A (2010) Coevolutionary games: a mini review. Biosystems 99(2):109-125

50. Pacheco JM, Santos FC, Souza MO, Skyrms B (2009) Evolutionary dynamics of collective action in n-person stag hunt dilemmas. Proc R Soc Lond B Biol Sci 276(1655):315-321

51. Sambandham M (1976) On the real roots of the random algebraic equation. Indian J Pure Appl Math 7(9):1062-1070

52. Sambandham M (1977) On a random algebraic equation. J Indian Math Soc 41(1-2):83-97

53. Sambandham M (1979) On the upper bound of the number of real roots of a random algebraic equation. J Indian Math Soc 42(1-4):15-26 1978

54. Sambandham M (1979) On the average number of real zeros of a class of random algebraic curves. Pacific J Math 81(1):207-215

55. Sandholm WH (2010) Population games and evolutionary dynamics. MIT Press, Cambridge

56. Sasaki T, Chen X, Perc M (2015) Evolution of public cooperation in a monitored society with implicated punishment and within-group enforcement. Sci Rep 5:1-12

57. Szolnoki A, Mobilia M, Jiang LL, Szczesny B, Rucklidge AM, Perc M (2014) Cyclic dominance in evolutionary games: a review. J R Soc Interface 11(100)

58. Szolnoki A, Perc M (2014) Coevolutionary success-driven multigames. EPL (Europhysics Letters) 108(2):28004

59. Szolnoki A, Perc M (2016) Biodiversity in models of cyclic dominance is preserved by heterogeneity in site-specific invasion rates. Sci Rep 6:1-9

60. Santos FC, Pinheiro FL, Lenaerts T, Pacheco JM (2012) The role of diversity in the evolution of cooperation. J Theor Biol 299:88-96

61. Stewart AJ, Parsons TL, Plotkin JB (2016) Evolutionary consequences of behavioral diversity. Proc Natl Acad Sci 113(45):E7003-E7009

62. Souza MO, Pacheco JM, Santos FC (2009) Evolution of cooperation under n-person snowdrift games. J Theor Biol 260(4):581-588

63. Schuster P, Sigmund K (1983) Replicator dynamics. J Theo Biol 100:533-538

64. Santos FC, Santos MD, Pacheco JM (2008) Social diversity promotes the emergence of cooperation in public goods games. Nature 454:213-216

65. Taylor PD, Jonker L (1978) Evolutionary stable strategies and game dynamics. Math Biosci 40:145-156

66. Tuyls K, Parsons S (2007) What evolutionary game theory tells us about multiagent learning. Artif Intell 171(7):406-416

67. Tao T, Vu V (2015) Local universality of zeroes of random polynomials. Int Math Res Notices 2015(13):5053

68. Wang X-S, Wong R (2012) Asymptotic of orthogonal polynomials via recurrence relations. Anal Appl 10(02):215-235

69. Zeeman EC (1980) Population dynamics from game theory. Lect Notes Math 819:471-497 